\title{
Erratum
}

\section{Chemical Bonding from Superposition of One-Center Charge Densities}

Ralph G. Pearson

Theoret. Chim. Acta (Berl.) 52, 253 (1979)

On page 255, the last line of paragraph 5 should read:

"For $\mathrm{H}_{2}$ the energy can be lowered by $0.68 \mathrm{eV}$ by floating 0.13 a.u."

Received November 20, 1979 